\title{
MODELO BIDIMENSIONAL SIMPLE PARA EL DESARROLLO DE UNA CORRIENTE DE TIPO DIPOLAR EN UN LÍQUIDO HOMOGÉNEO EN LA REGIÓN ECUATORIAL DEL OCÉANO
}

Valery Tchantsev, Orientador Científico, PhD. Oceanólogo, C.C.C.P

Anna Danshina, MSc. Oceanóloga, Universidad Hidrometeorológica del Estado Ruso, San Petersburgo

\section{esumen}

Se presenta un modelo matemático de formación de la corriente de tipo dipolar en un líquido homogéneo en la Región Ecuatorial del Océano. Se intenta describir la dependencia de coeficientes de viscosidad turbulenta de un gradiente de presión, gradiente de velocidad y módulo de velocidad de corriente. Finalmente, se presentan los resultados de experimentación numérica del desarrollo de una corriente de tipo dipolar en un líquido homogéneo.

\section{bstract}

Mathematical model of formation of dipolar types current in a homogeneous liquid of equatorial region of ocean is submitted. Attempt to describe dependence of turbulent viscosity coefficients from a gradient of pressure, gradient of speed and module of speed of current is made. Results of numerical experiment of development of dipolar types current in a homogeneous liquid are presented. 


\section{INTRODUCCIÓN}

Los resultados del procesamiento de imágenes de satélite de la superficie del océano muestran que las corrientes de tipo dipolar son una forma de movimiento universal de las aguas superficiales, son observados con alta frecuencia [Ginzburg, Fiodorov, 1984, 1984, 1988] y son conocidas como "Las corrientes de Hongo". La Corriente de "hongo" es especialmente una inestacionaria forma del movimiento. Representa un flujo estrecho, que presenta al final dos vórtices ciclónico y anticiclónico, incorporados en una estructura común. La formación y dinámica de los vórtices dipolares en un líquido homogéneo y estratificado han sido investigados con ayuda de modelación matemática y de laboratorio [Kozlov, Macarov, 1985, Voropaev, Filippov, 1985, Afanasiev, Voropaev, Potilitsin, 1994].

La base de este trabajo es la representación del proceso de formación de vórtices dipolares como forma análoga al plano de los anillos de vórtices formados en una explosión nuclear. Tales estructuras dinámicas son una consecuencia de alta concentración local de energía cinética, y la formación y distribución de una onda de presión, la cual conduce a la formación de movimientos ordenados de vórtices. En la modelación de la Corriente de "Hongo" creemos, que esta forma de circulación se presenta en una capa superficial delgada de espesor fijo.

\section{DESCRIPCIÓN FÍSICA DEL PROCESO}

En el líquido homogéneo en reposo se ubica una fuente emisora de impulso dirigido, la cual ocasiona el movimiento del líquido en dirección de la acción del impulso. El gradiente de velocidad generado crea un gradiente de presión, (grad $\boldsymbol{P})$ formando una onda de presión. Esta onda se mueve con la velocidad de fase en dirección del eje $\boldsymbol{X}$ y del eje $\boldsymbol{Y}$. La fuente activa de impulso adaptada en el modelo trabajó en forma permanente, produciendo, por lo tanto, un constante flujo de energía cinética que se extiende en todas las direcciones. $\mathrm{Al}$ accionar la fuente en el momento inicial, se genera el flujo de impulso. El flujo se dirige hacia el fluido en reposo, y una vez ocurre el encuentro, surge un frente de onda de presión como consecuencia del gradiente de presión formado por la diferencia de velocidades. La velocidad de fase de la fuente de onda es menor que la velocidad de chorro del flujo de impulso. Cuando el flujo de impulso alcanza al frente de onda (localizado entre el líquido en reposo y el flujo generado por la fuente), encuentra máxima resistencia en dirección inicial del flujo en comparación con la que encuentra en sentido perpendicular a este. Naturalmente el líquido comenzará a fluir en la dirección donde encuentra menor resistencia, en el cual el gradiente de presión es negativo o tiende a cero.

A medida que la onda de presión se va alejando de la fuente, en dirección de la acción de la fuente generadora de impulso, la velocidad de la corriente se va nivelando gradualmente, es decir, donde inicialmente habían valores de velocidad del fluido próximos a cero, ahora son cercanos al valor del flujo de impulso. Como el gradiente de presión se forma por la diferencia de velocidades, en los puntos en que la velocidad ha incrementado y se aproxima a la velocidad inicial, el gradiente de presión es cero. Alrededor de la corriente generada por el flujo de impulso, se ubica el frente de la onda de presión con valores de presión positivos. Como los fluidos circulan en sentido de mayor a menor gradiente de presión, el flujo cercano al frente de la onda de presión se dirigirá hacia la corriente del flujo de impulso, lo cual da lugar a la ocurrencia de los vórtices dipolares y a la formación de "La corriente de Hongo". Dos factores influyen en la formación de las estructuras de vórtice al final del flujo de impulso: la diferencia de presión y el carácter de mínima resistencia que ofrece el fluido en reposo en sentido perpendicular al Flujo.

\section{FÓRMULAS DEL MODELO}

La fuente del flujo de impulso se ubica en una capa superficial de poco espesor de lejos de la frontera del área de trabajo y en dirección norte. Los componentes horizontales de velocidad de corriente varían poco con la profundidad. El líquido tiene distribución homogénea de densidad. La rotación de la Tierra no afecta la formación de circulación del agua. El cambio de presión en la corriente ocurre como resultado del movimiento de la onda de presión a velocidad de fase y así mismo la variación de su velocidad. El resultado, un sistema de ecuaciones inestacionarias describe la corriente de tipo dipolar de modo horizontal bidimensional como se indica a continuación:

(1)

$\frac{\partial u}{\partial t}+u \frac{\partial u}{\partial x}+v \frac{\partial u}{\partial y}=-\frac{1}{\rho} \frac{\partial P}{\partial x}+\frac{\partial}{\partial x} K_{l x 1} \frac{\partial u}{\partial x}+\frac{\partial}{\partial y} K_{l x 2} \frac{\partial u}{\partial y}+\frac{\partial}{\partial y} K_{k x 3} \frac{\partial v}{\partial x}+\frac{\tau_{x}}{H}$ 
(2)

$$
\frac{\partial v}{\partial t}+u \frac{\partial v}{\partial x}+v \frac{\partial v}{\partial y}=-\frac{1}{\rho} \frac{\partial P}{\partial y}+\frac{\partial}{\partial y} K_{l y 1} \frac{\partial v}{\partial y}+\frac{\partial}{\partial x} K_{l y 2} \frac{\partial v}{\partial x}+\frac{\partial}{\partial x} K_{l y 3} \frac{\partial u}{\partial y}+\frac{\tau_{y}}{H}
$$

$$
\frac{\partial P}{\partial t}+u \frac{\partial P}{\partial x}+v \frac{\partial P}{\partial y}=-A_{p} \rho\left(u^{2} \frac{\partial u}{\partial x}+v^{2} \frac{\partial v}{\partial y}\right)
$$

donde $\boldsymbol{x}, \boldsymbol{y}$ - los ejes de coordenadas en dirección para el este y norte consiguientemente;

$$
\begin{aligned}
& \boldsymbol{P} \text { - la presión del agua; } \\
& \rho \text { - la densidad del agua; }
\end{aligned}
$$

$\boldsymbol{u}, \boldsymbol{v}$ - los componentes de velocidad de corriente promedio en el espesor de la capa;

$\boldsymbol{H}$ - el espesor de la capa;

$\tau_{x^{\prime}} \tau_{y}-$ los componentes de tensor de fricción sobre la frontera inferior de la capa;

$\boldsymbol{A} \boldsymbol{p}$ - la constante adimensional.

$\boldsymbol{K}_{l x p}, \boldsymbol{K}_{l x x^{2}}, \boldsymbol{K}_{l y,}, \boldsymbol{K}_{l y 2}, \boldsymbol{K}_{l x y}, \boldsymbol{K}_{l y 3}$ - los coeficientes de viscosidad turbulenta horizontal;

La dimensión del área de modelación empleada fue superior a la escala del fenómeno de modelación para excluir influencia de fronteras. En todas las fronteras se estableció la condición de corriente libre.

En la frontera inferior la condición de deslizable (sin fricción) está dada por las expresiones:

$$
\tau_{x}=\mathbf{0}, \tau_{y}=\mathbf{0} .
$$

La turbulencia en el campo de desarrollo de los movimientos ordenados del vórtice no es homogénea. La mayor resistencia del movimiento proviene de la dirección de acción del impulso. Para obtener el movimiento ordenado del vórtice en el modelo, se crearon las coeficientes de transmisión de derivadas mixtas $\left(\boldsymbol{K}_{l x 3}\right.$ y $\left.\boldsymbol{K}_{l y 3}\right)$. Los coeficientes de viscosidad turbulenta horizontal pueden cambiar desde cualquier valor mínimo (de viscosidad molecular) hasta cualquier valor máximo. Tal dependencia puede ser descrita por la función trigonométrica Arctg o función de potencia. Los coeficientes de viscosidad turbulenta horizontal están en función del gradiente de presión (grad $\boldsymbol{P})$. El valor del grad $\boldsymbol{P}$ puede ser negativo, por lo tanto para efecto de cálculos, es más conveniente usar una función trigonométrica. Los coeficientes en direcciones $\boldsymbol{X}$ y $\boldsymbol{Y}$ tienen la misma forma matemática. A continuación se presentan los coeficientes, así:
(4)

$K_{L \times 1}=K_{l}\left\{1+\frac{\operatorname{arctg}\left(m\left(\frac{\partial P}{\partial x}-j\right)\right)}{\pi / 2}\right\}$

$K_{t<2}=K_{l}\left\{1-\frac{\operatorname{arctg}\left(m\left(\frac{\partial P}{\partial x}-j\right)\right)}{\pi / 2}\right\}$

(6)

$K_{l}=K_{l 0}+l^{2}\left(\frac{\partial u}{\partial x}+\frac{\partial v}{\partial y}\right)$

(7)

$K_{L x 3}=F_{k x}\left\{1+\frac{\operatorname{arctg}\left(m\left(\frac{\partial P}{\partial y}-j\right)\right)}{\pi / 2}\right\}$

(8)

$F_{k x}=\widetilde{F}_{k x}\left\{1+\frac{\operatorname{arctg}\left(m\left|\frac{\partial y}{\partial y}\right|\right)}{\pi / 2}\right\}$

(9)

$\tilde{F}_{k x}=\frac{F_{v p}}{(0.001+0.01 V)^{2}} \frac{\partial v}{\partial y}$

donde $\boldsymbol{F}_{v \boldsymbol{p}}, \boldsymbol{j}, \boldsymbol{m}$ - las constantes dimensionales;

$\boldsymbol{l}$ - la escala de turbulencia horizontal proporcional al paso de espacio;.

$\boldsymbol{K} \boldsymbol{l} \boldsymbol{0}$ - el coeficiente de viscosidad molecular;

Para la solución del problema se usó el método de iteraciones. La influencia de grad $\boldsymbol{P}$ en la formación de "La Corriente de Hongo" es obvia. Pero $\boldsymbol{K}_{l x 3}$ y $\boldsymbol{K}_{l y 3}$ dependen aún del módulo y gradiente de velocidad (Fig. 1). Al acercarse al frente de presión, la velocidad de corriente disminuye rápidamente, pero el coeficiente de viscosidad turbulenta de la derivada compuesta aumenta. Los parámetros en (7) se forman así que se incremente $\boldsymbol{K}_{l x 3}$ y $\boldsymbol{K}_{l y 3}$ en el campo positivo de grad P. Para obtener el movimiento del líquido a lo largo del frente de presión, el valor máximo del 


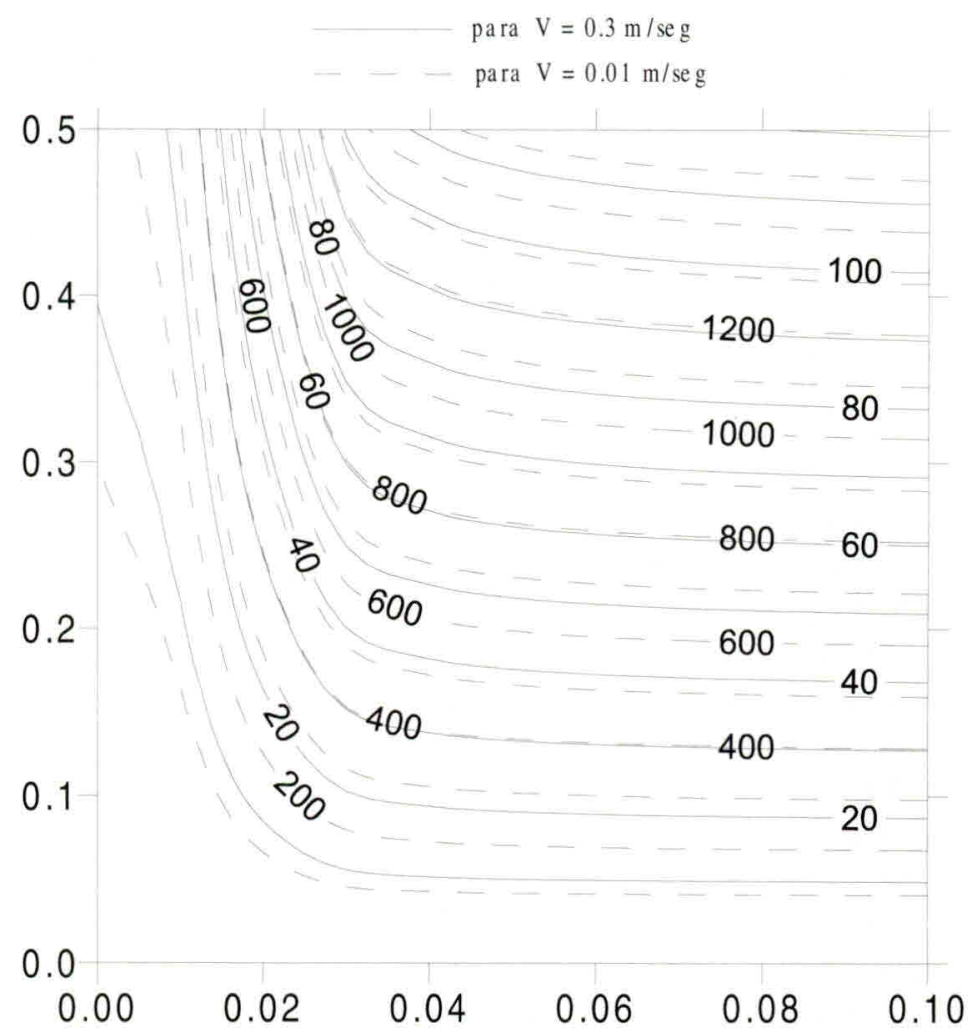

Figura 1: Coeficiente de transmisión de derivada mixta $\left(\mathbf{K}_{\mathrm{lx} 3}\right)$ en función de gradiente de presión y velocidad y módulo de velocidad

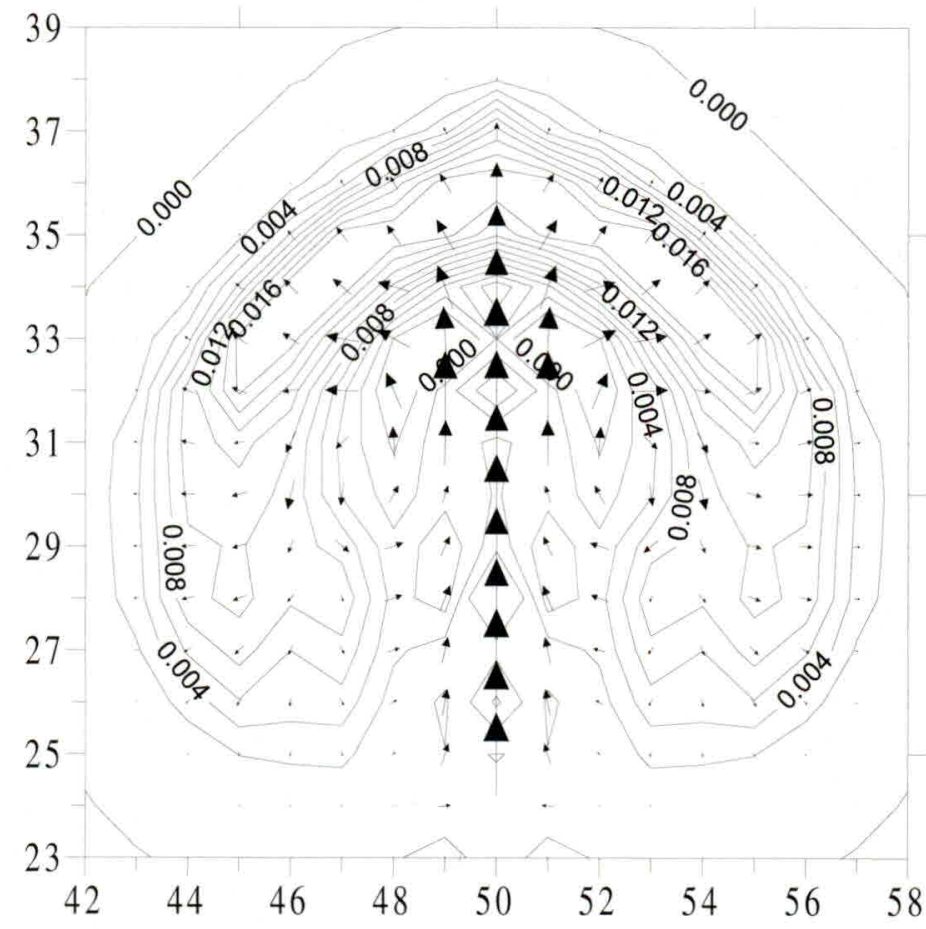

Figura 2: Distribución de presión del agua y velocidad después de 5 horas de comenzar el experimento 
gradiente de presión debe coincidir con el valor máximo del coeficiente de viscosidad turbulenta de la derivada compuesta. La onda de presión divide el área de modelación en dos partes, la interna debe tener un coeficiente mucho mayor que el de la externa.

\section{DESCRIPCIÓN DE EXPERIMENTACIÓN NUMÉRICA}

La región de modelación tiene un área de 50 por 50 kilómetros. La solución del modelo estuvo sobre una grilla de 500 por 500 metros. El intervalo de integración en el tiempo es 100 segundos. En el momento inicial del tiempo, el agua está en reposo en la región de modelación. La fuente de impulso está en la parte del fondo (sur) del área de estudio y funciona durante toda la experimentación en dirección norte. La velocidad de la corriente en la fuente es $0.5 \mathrm{~m} /$ seg. Después de 5 horas de comenzar el experimento, a una distancia de $7000 \mathrm{~m}$ de la fuente se forma un vórtice de dipolo (Fig. 2). Su tamaño y la distribución de la velocidad corresponde a "La Corriente de Hongo" que se observa en el Océano.

\section{CONCLUSIONES}

Se hizo una aproximación a los coeficientes de viscosidad turbulenta horizontal. Se dan a conocer las etapas principales de evolución de los vórtices dipolares con base en experimentaciones numéricas. Así, el modelo determinado describe cualitativamente las características principales de "Las Corrientes de Hongo" naturales.

\section{Bibliografía}

GINZBURG A, FIODOROV K. Capa superficial del océano. Leningrado, Gidrometeoizdad, 1988, 300 p.

GINZBURG A FIODOROV K. Corrientes de tipo "hongo" de Océano. Moscú, Investigaciones de Tierra del cosmos, 1984, №3, pp.18-25

GINZBURG A, FIODOROV K. Evolución de las corrientes de tipo "hongo" en el Océano. Moscú, Informe de Academia de Ciencia de U.R.S.S., 1984, V. 276, №2, pp. 481-484

KOZLOV V., MACAROV V. Modelo hidrodinámico de formación de corrientes de tipo "hongo" en el Océa- no. Moscú, Informe de Academia de Ciencia de U.R.S.S., 1985, V. 281, ? 5, pp.1213-1215

VOROPAEV S., FILIPPOV I. Desarrollo de un chorro horizontal en líquido de densidad homogéneo y de estratificado. Experimenta laboratorio. Noticias de Academia de Ciencia de U.R.S.S., FAO, 1985, V. 21, №9, pp. $964-972$

AFANASIEV Ya., VOROPAEV S., POTILITSIN P. Interacción de vóitices de dipolo: la teoría, el experimenta laboratorio. Noticias de Academia de Ciencia de U.R.S.S., FAO, 1994, V. 30, № 5, pp. 696-703. 